\title{
ON THE MAXIMUM MASS AND MINIMUM ROTATION PERIOD OF RELATIVISTIC UNIFORMLY ROTATING WHITE DWARFS
}

\author{
KUANTAY BOSHKAYEV* ${ }^{*}$, JORGE RUEDA ${ }^{\dagger}$ and REMO RUFFINI ${ }^{\ddagger}$ \\ Dipartimento di Fisica, Università di Roma La Sapienza \\ Piazzale Aldo Moro 5, I-00185 Roma, Italy \\ ICRANet, Piazzale della Repubblica 10, I-65122 Pescara, Italy \\ *kuantay@icra.it \\ †jorge.rueda@icra.it \\ ${ }_{\text {‡ruffini@icra.it }}$
}

\begin{abstract}
We investigate the stability of general relativistic uniformly rotating white dwarfs against secular and dynamical instabilities. We determine the minimum rotation period of stable white dwarfs depending on chemical composition of the white dwarf matter taking into account the Coulomb interactions as well as the nuclear interactions and the electroweak equilibrium at high densities, within the relativistic Feynman-Metropolis-Teller equation of state.
\end{abstract}

Recently, equilibrium configurations of non-rotating (static) ${ }^{4} \mathrm{He},{ }^{12} \mathrm{C},{ }^{16} \mathrm{O}$ and ${ }^{56} \mathrm{Fe}$ white dwarfs (WDs) within general relativity (GR) have been constructed in Ref. 1 . The white dwarf matter has been there described by the relativistic generalization of the Feynman-Metropolis-Teller (RFMT) equation of state (EoS) obtained by Rotondo et $a .^{2}$ A new mass-radius relation that generalizes both the works of Chandrasekhar ${ }^{3}$ and Hamada \& Salpeter ${ }^{4}$ has been there obtained, leading to a smaller maximum mass and a larger minimum radius with respect to the previous calculations. In addition, it has been shown how both GR and inverse $\beta$-decay are relevant for the determination of the maximum stable mass of non-rotating WDs.

It is therefore of interest to generalize the above results to the case of rotation. As a first attempt, we constructed in Ref. 5 general relativistic uniformly rotating WDs in the simplified case when microscopic Coulomb screening is neglected in the EoS, following the Chandrasekhar ${ }^{3}$ approximation by describing the matter as a locally uniform fluid of electrons and nuclei. The average molecular weight in the Chandrasekhar EoS is $\mu=A / Z$, where $A$ is the mass number and $Z$ is the number of protons in a nucleus.

As a second attempt, in Ref. 6 we calculated the maximum mass of rotating ${ }^{4} \mathrm{He},{ }^{12} \mathrm{C},{ }^{16} \mathrm{O}$ and ${ }^{56} \mathrm{Fe}$ WDs using the Salpeter ${ }^{7}$ and the RFMT EoS. As a result we obtained there different maximum mass for different chemical composition of WD matter. 
In this work we apply Hartle's formalism to the description of the structure of rotating objects, up to second order terms in the angular velocity of the star $\Omega$. In this "slow" approximation regime, the solution of the Einstein equations in the exterior can be written in analytic closed form in terms of the mass $M$, angular momentum $J$ and quadrupole moment $Q$ of the rotating star, by perturbing the corresponding seed static solution. The interior solution, which matches with the exterior one, can be then constructed by solving numerically a system of ordinary differential equations. ${ }^{8,9}$

The parameters $M, J$ and $Q$, are obtained for a given EoS, from the matching procedure between the internal and external solutions. Note, that the total mass is defined by $M=M^{J \neq 0}=M^{J=0}+\delta M$, where $M^{J=0}$ is the mass of a static white dwarf with the same central density as $M^{J \neq 0}$, and $\delta M$ is the contribution to the mass due to rotation.

The main objective of this work is to answer the questions whether the maximum mass of uniformly rotating WDs is stable or not and what their minimum rotation period is.

There are two main stability criteria for non-rotating WDs: the well-known general relativity (GR) instability and inverse $\beta$-decay instability. The inverse $\beta$-decay instability is related to the fact that with the increasing mass of WDs the central density will increase, hence the electrons will become ultra relativistic and their energy will increase until it reaches a certain value of the Fermi energy (depending on chemical composition of a WD matter) which allows the electrons to be captured by the protons inside the nuclei, forming neutrons. This process is called inverse $\beta$ decay or neutronization and is written in a compact form $p+e^{-} \rightarrow n+\nu_{e}$, where $p$ is the proton, $e^{-}$is the electron, $n$ is the neutron and $\nu_{e}$ is the electron neutrino. Such a process conserves the number of baryons creating neutron rich nuclei through $e^{-}$ capture. Due to the fact that most of the pressure of the WD is supported by the electrons, the inverse $\beta$-decay process leads to the pressure decrease of the WD by increasing the density which causes the instability of the star.

For rotating WDs there exist additional stability criteria. One of them is the mass shedding limit. When a star spins so rapidly the centrifugal forces will prevail over gravitational force and the star will start losing its matter on the equatorial plane. ${ }^{11}$ The procedure of estimating the mass shedding angular velocity was developed in Friedman et al. ${ }^{12}$

In addition to the mass shedding limit there are two more stability criteria called secular (axisymmetric) and dynamical instabilities. Usually in the literature the conditions $T / W=0.14, e=0.81267$ and $T / W=0.25, e=0.95288$ are adopted as the upper limit for the onset of the secular and dynamical instabilities, ${ }^{10}$ where $T / W$ is the rotational/gravitational binding energy and $e$ is the eccentricity of rotating objects. These values are absolute upper limits for the ideal case of Maclaurin spheroids ${ }^{10}$ and in practice, they can not be applied to objects like uniformly rotating WDs since they have smaller $T / W$ and $e$ with respect to the Maclaurin spheroids (for details see Fig. 1). However Friedman et. al. ${ }^{13}$ developed the turning 

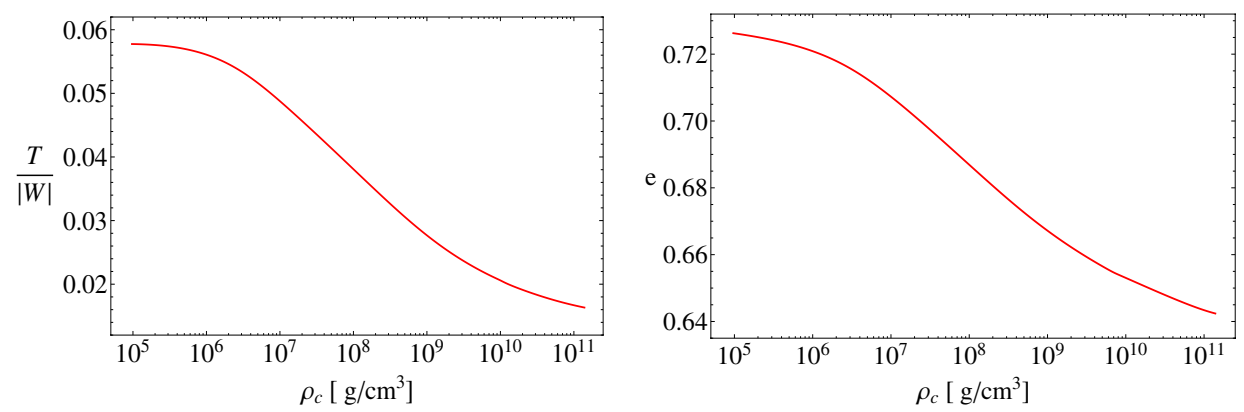

Fig. 1. Left panel: $T /|W|$ is the rotational/gravitational energy versus $\rho_{c}$. Right panel: $e$ is the quadrupole eccentricity versus $\rho_{c}$, along the Keplerian sequence for ${ }^{4} \mathrm{He}$ WD with the RFMT EoS.

point method of axisymmetric (secular) instability for uniformly rotating stars. The main idea of the turning point method is that along a sequence of constant angular momentum $J=$ const the condition $(\partial M(\rho, J) / \partial \rho)_{J}=0$ separates secularly stable and unstable stars. For $J=0$ we have the condition for the maximum mass of nonrotating WDs $\partial M(\rho) / \partial \rho=0$. Moreover it was shown in Friedman et. al. ${ }^{13}$ that a sequence of uniformly rotating stars becomes dynamically unstable after it has become secularly unstable. That is, for fixed angular momentum the point of secular instability occurs at lower central density with respect to dynamical instability.

We found that along the Keplerian (mass shedding) sequence there are no maxima of $J=$ const sequences. It means that along the Keplerian sequence all uniformly rotating WDs are secularly, hence dynamically stable. For this reason we increase the central density all the way up to inverse $\beta$-decay density in order to define the minimum rotation period of WDs, since this is the only limitation which guarantees the stability before the trigger of gravitational collapse. Thus, the minimum period of WDs is determined along the Keplerian sequence at the critical central density for inverse $\beta$-decay instability.

In Fig. 1 the ratio between the rotational energy and gravitational energy versus the central density (left panel) and the eccentricity versus the central density (right panel) are shown. With the increasing central density these ratios start to decrease, which shows that the system is gravitationally bound and secularly stable.

In Table 1 the maximum mass of rotating WDs is shown for different EoSs and different chemical composition of WD matter. It is interesting to compare the above results with the classic work of Roxburgh and Durney. ${ }^{14}$ They found a maximum mass of WDs $1.4825 M_{\odot}$ for the mass-shedding sequence, as well as the critical polar radius $363 \mathrm{~km}$ for rotating instability, for the Chandrasekhar EoS with $\mu=2$ (see Table 1 ). The Roxburgh critical radius is rather small with respect to our critical polar radii. It is clear that such a small radius would lead to a configuration with central density over or very close to the limit established by inverse $\beta$-decay: the average density obtained for the Roxburgh's critical configuration is $\sim 1.46 \times$ 
Table 1. The maximum mass and corresponding critical density for the general relativistic uniformly rotating ${ }^{4} \mathrm{He},{ }^{12} \mathrm{C},{ }^{16} \mathrm{O}$ and ${ }^{56} \mathrm{Fe}$ WDs with different EoS. The parameters $R_{p o l}, R_{e q}$ and $P$ are the polar, the equatorial radii and the rotation period of WDs, respectively.

\begin{tabular}{lcccccc}
\hline Comp. & EoS & $\rho_{\text {crit }}\left(\mathrm{g} / \mathrm{cm}^{3}\right)$ & $R_{\text {pol }}(\mathrm{km})$ & $R_{e q}(\mathrm{~km})$ & $M_{\max }^{J \neq 0} / M_{\odot}$ & $P(\mathrm{sec})$ \\
\hline$\mu=2$ & Chandra & $1.07 \times 10^{10}$ & 1198.91 & 1583.47 & 1.5159 & 0.884 \\
\hline \multirow{2}{*}{${ }^{4} \mathrm{He}$} & Salpeter & $1.07 \times 10^{10}$ & 1193.08 & 1575.94 & 1.4996 & 0.883 \\
\hline \multirow{2}{*}{${ }^{12} \mathrm{C}$} & RFMT & $5.46 \times 10^{9}$ & 1458.58 & 1932.59 & 1.5001 & 1.199 \\
\hline \multirow{2}{*}{${ }^{16} \mathrm{O}$} & Salpeter & $1.08 \times 10^{10}$ & 1183.99 & 1564.16 & 1.4833 & 0.878 \\
& RFMT & $6.95 \times 10^{9}$ & 1349.15 & 1785.98 & 1.4736 & 1.074 \\
\hline \multirow{2}{*}{$56 \mathrm{Fe}$} & Ralpeter & $1.09 \times 10^{10}$ & 1178.88 & 1556.68 & 1.4773 & 0.875 \\
& RFMT & $7.68 \times 10^{9}$ & 1308.09 & 1730.65 & 1.4667 & 1.027 \\
\hline
\end{tabular}

Table 2. The minimum rotation period of WDs. Critical density for inverse $\beta$ decay and corresponding mass for the general relativistic rotating ${ }^{4} \mathrm{He},{ }^{12} \mathrm{C},{ }^{16} \mathrm{O}$ and ${ }^{56} \mathrm{Fe}$ WDs. The parameters $R_{p o l}, R_{e q}$ and $P$ are the polar, the equatorial radii and the rotation period of WDs, respectively.

\begin{tabular}{lcccccc}
\hline Comp. & EoS & $\rho_{\text {crit }}^{\beta}\left(\mathrm{g} / \mathrm{cm}^{3}\right)$ & $R_{p}(\mathrm{~km})$ & $R_{e q}(\mathrm{~km})$ & $M^{J \neq 0} / M_{\odot}$ & $P_{\min }(\mathrm{sec})$ \\
\hline$\mu=2$ & Chandra & $1.37 \times 10^{11}$ & 562.79 & 734.54 & 1.4963 & 0.281 \\
\hline \multirow{2}{*}{${ }^{4} \mathrm{He}$} & Salpeter & $1.37 \times 10^{11}$ & 560.41 & 731.51 & 1.4803 & 0.281 \\
\hline \multirow{2}{*}{${ }^{12} \mathrm{C}$} & RFMT & $1.39 \times 10^{11}$ & 563.71 & 735.55 & 1.4623 & 0.285 \\
\hline \multirow{2}{*}{${ }^{16} \mathrm{O}$} & Salpeter & $3.88 \times 10^{10}$ & 815.98 & 1070.87 & 1.4775 & 0.498 \\
\hline \multirow{2}{*}{${ }^{5} \mathrm{~F} \mathrm{Fe}$} & Salpeter & $1.89 \times 10^{10}$ & 1005.62 & 1324.43 & 1.4761 & 0.686 \\
\hline
\end{tabular}

$10^{10} \mathrm{~g} / \mathrm{cm}^{3}$, very close to $\rho_{\text {crit }}^{\beta}=3.97 \times 10^{10} \mathrm{~g} / \mathrm{cm}^{3}$ for ${ }^{12} \mathrm{C}$ WDs and even closer to $\rho_{\text {crit }}^{\beta}=1.94 \times 10^{10} \mathrm{~g} / \mathrm{cm}^{3}$ for ${ }^{16} \mathrm{O}$ WDs. ${ }^{1}$ In Table 2 the minimum rotation period of uniformly rotating WDs is shown for different EoS and different chemical composition of WD matter.

We have here computed general relativistic configurations of uniformly rotating WDs using a realistic description of a WD which takes into account the specific chemical composition, the Coulomb interactions, as well as the nuclear interactions and the electroweak equilibrium at high densities within the RFMT EoS, extending the recent results on non-rotating WDs. ${ }^{1}$ We have shown, in particular, that the maximum mass of rotating WDs is stable against secular and dynamical instabilities and determined the minimum rotation period of rotating WDs.

These results presented here play a fundamental role in the determination of the entire region of stability of rotating WDs. 


\section{References}

1. M. Rotondo, J. A. Rueda, R. Ruffini and S.-S. Xue, Phys. Rev. D 84 (2011) 084007.

2. M. Rotondo, J. A. Rueda, R. Ruffini and S.-S. Xue, Phys. Rev. C 83 (2011) 045805.

3. S. Chandrasekhar, ApJ $\mathbf{7 4}$ (1931) 81.

4. T. Hamada and E. E. Salpeter, ApJ 134 (1961) 683.

5. K. Boshkayev, J. Rueda and R. Ruffini, IJMPE 20 (2011) 136.

6. K. Boshkayev, J. Rueda and R. Ruffini, in From Nuclei to White Dwarfs to Neutron Stars, ed. (A. Mezzacappa and R. Ruffini, 2011)

7. E. E. Salpeter, ApJ 134 (1961) 669.

8. J. B. Hartle, Ap.J. 150 (1967) 1005.

9. J. B. Hartle and K. S. Thorne, ApJ 1531968807.

10. S. Chandrasekhar, Ellipsoidal Figures of Equilibrium, (New Haven and London, Yale University Press, 1969)

11. N. Stergioulas Living Rev. 7 (2003) 3.

12. J. L. Friedman, J. R. Ipser and L. Parker, ApJ 304 (1986) 115.

13. J. L. Friedman, J. R. Ipser and R. D. Sorkin, ApJ 325 (1988) 722.

14. I. W. Roxburgh and B. R. Durney, Zeitschrift fur Astrophysik 64 (1966) 504. 